\title{
Microbiome and Antimicrobial Sensitivity Pattern of Endotracheal Secretions in Mechanically Ventilated Patients in ICUs in a Tertiary Care Hospital
}

\author{
Sharabu Yamini ${ }^{1}$, Rangineni Jayaprada르. Chejarla Tirumala ${ }^{3}$, Aloka Samantaray ${ }^{4}$, Banda Venkata Ramana ${ }^{5}$
}

1, 2, 3, 4, 5 Department of Microbiology, Sri Venkateswara Institute of Medical Sciences, Tirupati, Andhra Pradesh, India.

\section{ABSTRACT}

\section{BACKGROUND}

Mechanical ventilation is a life-saving procedure for most patients in ICUs. But it has a risk of acquiring respiratory tract infections resulting in high morbidity and mortality. If not treated early, it may lead to ventilator-associated pneumonia (VAP). Early diagnosis and appropriate antibiotic therapy at right time in the right dosage is essential for better clinical outcome. The objectives of the study were to analyse the microbiomes of endotracheal samples and their antimicrobial susceptibility pattern in mechanically ventilated patients in ICUs and to frame an institution based local antibiotic policy especially for them.

\section{METHODS}

A retrospective study was conducted in a tertiary care hospital and sample data was collected which included all adult patients who were mechanically ventilated for various co-morbid conditions in ICUs from January 2019 to December 2019. All the samples were processed for microscopy, culture and antimicrobial susceptibility pattern.

\section{RESULTS}

Among 848 endotracheal samples, 673 (79.4\%) samples were culture positive and $175(20.6 \%)$ samples were sterile. Most common organism was Acinetobacter spp $(\mathrm{N}=240)$ followed by Klebsiella spp $(\mathrm{N}=225)$ and Pseudomonas spp $(\mathrm{N}=97)$. In our study, polymicrobial infections were $186(21.7 \%)$. Sixty-eight percent of organisms were multidrug-resistant. Carbapenem resistance was $58 \%$.

\section{CONCLUSIONS}

The most common complication of mechanical ventilation is an endotracheal infection which in turn leads to prolonged stay in hospital, morbidity and mortality. It is essential to know about the local microbiome and its antibiotic susceptibility pattern.

\section{KEY WORDS}

Microbiome-human, Antibiogram, Endotracheal Intubation.
Corresponding Author:

Dr. Rangineni Jayaprada, Associate Professor, Department of Microbiology, SVIMS, Tirupathi-517501,

Andhra Pradesh, India.

E-mail: pradarangineni@yahoo.com

DOI: $10.14260 /$ jemds/2022/16

How to Cite This Article:

Yamini S, Jayaprada $R$, Tirumala $C$, et al. Microbiome and antimicrobial sensitivity pattern of endotracheal secretions in mechanically ventilated patients in ICUs in a tertiary care hospital. J Evolution Med Dent Sci 2022;11(01):83-87, DOI: 10.14260/jemds/2022/16

Submission 02-12-2021, Peer Review 27-12-2021, Acceptance 04-01-2022, Published 25-01-2022.

Copyright (C) 2022 Sharabu Yamini et al. This is an open access article distributed under Creative Commons Attribution License [Attribution 4.0 International (CC $B Y 4.0)]$ 


\section{BACKGROUND}

According to the surveillance data from the National Nosocomial Infections Surveillance (NNIS) system of the Centre for Disease Control and Prevention (CDC), 'Healthcare-associated pneumonia (HCAP) or Nosocomial pneumonia' is the most common infection in the intensive care units (ICUs).1,2 Mechanical ventilation (MV) is a lifesaving procedure for most of the patients in ICUs, but it is associated with a greater risk of acquiring respiratory tract infections resulting in high morbidity and mortality in critically ill patients. Mechanical ventilation is the artificial respiration of the lungs by using a mechanical ventilator to deliver oxygen to the lungs. Oxygen masks like negative pressure ventilation (NIV), non-invasive positive pressure ventilation (NIPPV), continuous positive pressure airway pressure (CPAP) and bi-level positive airway pressure (BiPAP) have been used for non-invasive ventilation in appropriately selected conscious patients.

Mechanical ventilation is usually indicated in physiologic impairment of the lung prophylactically. Mechanical ventilation serves only to assist breathing, still, there are certain potential complications which include pneumothorax, injury to the airway, alveolar damage, ventilator-associated pneumonia (VAP), and ventilator-associated tracheobronchitis. ${ }^{3,4}$

The occurrence of HCAP is most frequent in intubated patients with mechanical ventilation. ${ }^{5}$ The incidence of HCAP varies from 9 to $78 \%$, depending on the severity of illness, type of patients, techniques \& criteria used to diagnose pneumonia. ${ }^{6}$ Ventilator-associated pneumonia (VAP) is a lung infection that develops in a person who is on a ventilator after 48 hours of hospital admission. As per centre for disease control (CDC) guidelines, it is defined as ventilator-associated events (VAE). VAEs are defined by increase in oxygen $(\geq 0.2$ in FiO2) or positive end-expiratory pressure (PEEP) $(\geq 3 \mathrm{~cm}$ $\mathrm{H} 2 \mathrm{O}$ ), after a previous stable baseline of at least 2 days. There are three definition tiers within the VAE algorithm: 1) Ventilator-associated condition (VAC); 2) Infection-related ventilator-associated complication (IVAC), and 3) Possible VAP (PVAP). VAE is estimated to occur in 9-27 \% of all mechanically ventilated patients, with the highest risk being early in the course of hospitalization. ${ }^{7}$ The mortality rate in VAP ranges from $24 \%$ to $80 \%$ in several studies with a 2 to 10 fold higher risk of death rate in ICU ventilated patients. ${ }^{8}$

Respiratory tract infections especially in patients on ventilators and who are critically ill are associated with high morbidity and mortality. Early diagnosis and appropriate antibiotic therapy at right time are essential for a better clinical outcome. Patients who are mechanically ventilated are further at risk of acquiring respiratory tract infections, which may lead to VAP. The etiologic agents vary according to the number of patients in an ICU, duration of hospital stay, pre-existing illness and prior antimicrobial therapy. To start an empiric antibiotic therapy, knowledge of the local microbiome and its susceptibility pattern are quite essential and need to be analysed regularly to frame an institution based antibiotic policy periodically and made available to all clinicians involved in patient care, hence this study helps initiate an empirical antibiotic therapy in mechanically ventilated patients.

\section{Objectives}

1. To analyse the microbiome of endotracheal samples [endotracheal aspirates (ETAs) and endotracheal tube secretions (ETTs)] and their antimicrobial susceptibility pattern in mechanically ventilated patients in ICUs.

2. To frame an institution based local antibiotic policy especially for mechanically ventilated patients.

\section{METHODS}

A retrospective study was conducted in a tertiary care hospital and sample data was collected which included all adult patients who were mechanically ventilated for various comorbid conditions in ICUs from January 2019 to December 2019. All the endotracheal samples submitted were processed under aseptic precautions for aerobic culture on Nutrient agar, Blood agar and MacConkey agar within 2 hours of collection. Bacterial identification was based on their colony morphology, gram staining, motility and biochemical reactions by following standard microbiological techniques as per standard protocol.9,10 Antibiotic susceptibility testing was done by Kirby Bauer disk diffusion method on Mueller Hinton Agar (Oxoid, UK) as per clinical laboratory standard institute (CLSI) guidelines 2019.11

The zone diameter of inhibition was interpreted according to CLSI 2019 standards. Media (HiMedia, Mumbai) and disks (HiMedia, Mumbai) were checked for quality by using standard American Type Cell Culture (ATCC) strains Escherichia coli (ATCC 25922) and Staphylococcus aureus (ATCC 29213). The study was conducted after obtaining approval from Institutional Ethics Committee (IEC No: 1088).

\section{Statistical Analysis}

All the details regarding demographic characteristics i.e., hospital ID, age, gender, organism isolated and antibiotic sensitivity pattern were recorded and entered in Microsoft excel sheet and analyzed. The categorical variables were represented as percentages. The data were analyzed using SPSS (version 21).

\section{RESULTS}

Eight hundred and forty-eight (848) endotracheal sample data was collected. Among all endotracheal samples, 673 (79.4\%) samples showed culture positivity with significant colony count $\left(>10^{5} \mathrm{CFU} / \mathrm{ml}\right)$ after $24-48$ hours of aerobic incubation at $37^{\circ} \mathrm{C}$ and 175 (20.6\%) samples were sterile after 48 hours of aerobic incubation at $37^{\circ} \mathrm{C}$ from all mechanically ventilated patients from various ICUs. Male (66 $\%)$ predominance was observed when compared to females (34\%). Among them, the culture positivity rates in males and females were $80 \%$ and $78 \%$ respectively.

\begin{tabular}{|ccc|}
\hline $\begin{array}{c}\text { Total } \\
\text { Samples }\end{array}$ & $\begin{array}{c}\text { No. of Samples- Culture } \\
\text { Positive N (\%) }\end{array}$ & $\begin{array}{c}\text { No of Samples-Culture } \\
\text { Negative N (\%) }\end{array}$ \\
\hline 848 & $673(79.4 \%)$ & $175(20.6 \%)$ \\
\hline Table 1. Percentage of Culture Positivity among ET Samples: \\
\hline
\end{tabular}


Among all isolated organisms, 240 (28 \%) were Acinetobacter spp followed by 225 (26.5 \%) Klebsiella spp, 161 (19\%) Pseudomonas spp, 97 (11.5 \%) Escherichia spp, 51 (6 \%) enterobacter spp, 38 (4.5\%) Non fermenting Gramnegative bacilli (NFGNB). Percentage of polymicrobial infections was $21.9 \%$ in our study.

\begin{tabular}{|cc|}
\hline Organisms Isolated & Percentage \% (N) \\
Acinetobacter & $28(240)$ \\
Klebsiella spp & $26.5(225)$ \\
Pseudomonas & $19(161)$ \\
Escherichia species & $11.5(97)$ \\
Enterobacter spp & $6(51)$ \\
NFGNB & $4.5(38)$ \\
Staph aureus & $3.8(32)$ \\
Providencia & $3(26)$ \\
Citrobacter & $2.2(19)$ \\
Proteus spp & $1.4(12)$ \\
Coagulase-negative staphylococcus & $1.3(11)$ \\
Beta haemolytic staphylococcus & $0.8(7)$ \\
Candida & $0.4(4)$ \\
Aspergillus & $0.1(1)$ \\
Alpha haemolytic streptococci & $0.1(1)$ \\
Burkholderia cepacia complex & $0.1(1)$ \\
Moraxella & $0.1(1)$ \\
Total & $\mathbf{1 0 0}(673)$ \\
\hline Table 2. Percentage Distribution of Microorganisms Isolated in \\
Culture from Endotracheal Samples of Mechanically Ventilated \\
\hline
\end{tabular}

Among all endotracheal samples, culture positivity was seen accordingly in neurosurgery (97.2 \%) followed by respiratory ICU (RICU) (88.5\%), surgical oncology (88\%), nephrology (87.5 \%), general medicine (86\%), radiation oncology (83.3\%), neurology (82.4\%), emergency medicine (76\%) and cardiothoracic and vascular surgery (57 \%).

\begin{tabular}{|ccc|}
\hline Department & $\begin{array}{c}\text { Total } \\
\text { No. of Cases }\end{array}$ & $\begin{array}{c}\text { Percentage of Culture } \\
\text { Positivity (N) }\end{array}$ \\
Radiation oncology & 06 & $83.3(5)$ \\
RICU & 184 & $88.5(162)$ \\
Neurosurgery & 36 & $97.2(35)$ \\
General Medicine & 83 & $86(72)$ \\
Neurology & 51 & $82.4(42)$ \\
Emergency medicine & 380 & $76(291)$ \\
Nephrology & 08 & $87.5(7)$ \\
CT surgery & 70 & $57(40)$ \\
General surgery & 10 & $50(5)$ \\
Surgical oncology & 18 & $88(16)$ \\
Endocrinology & 1 & 0 \\
Cardiology & 2 & 0 \\
Surgical gastroenterology & 2 \\
\hline Table 3. Percentage and Department Wise Incidence of Infection in \\
Mechanically Ventilated Patients \\
\hline
\end{tabular}

In our study, resistance to penicillin group was $87.5 \%$ $(\mathrm{N}=28)$, resistance to cefoxitin was $40.6 \%(\mathrm{~N}=13)$ and inducible clindamycin resistance was $50 \%(\mathrm{~N}=16)$ among 32 isolates of $S$. aureus. No linezolid resistance was observed. All gram-positive organisms were sensitive to vancomycin.

Among all gram-negative bacilli $(\mathrm{N}=873), 77 \%(\mathrm{~N}=672)$ of organisms were multidrug-resistant. Extended spectrum beta lactamase (ESBL) organisms were $60 \%(N=523)$. In the case of aminoglycoside resistance, amikacin, gentamicin and netilmicin resistance was $45.6 \%, 48 \%$ and $43.6 \%$ respectively. Among all gram-negative bacilli, imipenem resistance was $48 \%$, meropenem resistance was $48 \%$ and ertapenem resistance was $58 \%$. The majority of the Enterobacteriaceae organisms were sensitive to polymyxin B. In all gram-negative bacilli, resistance to tigecycline $(\mathrm{N}=23)$ was observed and being $65.2 \%$ in acinetobacter spp, $17.4 \%$ in Klebsiella spp, $4.3 \%$ in citrobacter spp, $4.3 \%$ in Escherichia spp, $4.3 \%$ in Non-fermenting gram-negative bacilli and $4.3 \%$ in Providencia spp.

In acinetobacter spp, amikacin, gentamicin, netilmicin resistance was $61.7 \%, 61 \%$ and $53.8 \%$ respectively. Carbapenem resistance when individualized, imipenem and meropenem resistance was $70.4 \%$ and $77.5 \%$ respectively and ertapenem was very high and it was $93.3 \%$ in Acinetobacter spp which was noticed in our study. Fluoroquinolone resistance was $80 \%$ in acinetobacter spp.

In our study, isolated Escherichia spp were 97, percentage of resistance of amikacin was $25.8 \%$, gentamicin $35 \%$, netilmicin $28.9 \%$, imipenem $39 \%$, meropenem $12.4 \%$, ertapenem $48.5 \%$, cefazolin $80 \%$ and levofloxacin $73 \%$.

Isolated Klebsiella spp were 225 , the resistance of amikacin was $58 \%$, gentamicin was $60 \%$, netilmicin was 58 $\%$, imipenem was $56.4 \%$, meropenem was $57 \%$, ertapenem was $72 \%$, cefazolin was $91 \%$ and levofloxacin resistance was $71 \%$ as observed in our study.

In our study, Pseudomonas isolates were 161, the percentage of resistance of amikacin was 21 , gentamicin was $29 \%$, netilmicin was $25.5 \%$, imipenem was $28.6 \%$, meropenem $19.3 \%$, ceftazidime resistance $44 \%$, ciprofloxacin resistance $38.5 \%$ and piperacillin-tazobactam was $18 \%$.

\section{DISCUSSION}

Endotracheal intubation and mechanical ventilation are lifesaving procedures, essential in acutely critical clinical conditions like acute respiratory distress syndrome (ARDS), neurological diseases, trauma, sepsis etc. But there is a greater chance of acquiring infection after endotracheal intubation or mechanical ventilation. The percentage of infection rate in males was 80 and in females 78.7. A high percentage of infection rates in males may be due to increased sample size from male patients. We observed that there is no correlation between male gender and culture positivity rate. Variation in infection rates is highly dependent on infection prevention and control measures, pre-existing lung diseases, length of hospital stay or other risk factors like smoking and substance abuse. ${ }^{12}$

Mechanical ventilation is indicated to prevent death due to respiratory failure and also has the great disadvantage of causing lung infections after intubation. This respiratory tract infection after intubation can be either by the exogenous or endogenous route and finally may result in VAP.

Patients below 18 years of age were excluded from the study. The majority of the patients were reported in the age group between 40 and 80 yrs. Males contributed $66 \%$ and females contributed $34 \%$. In culture positivity, no age preponderance was observed in our study and infection could be dependent on pre-existing disease and infection prevention control practices.

Most of the samples were received from emergency medicine $(\mathrm{N}=380)$ followed by respiratory ICU $(\mathrm{N}=185)$ and general medicine $(\mathrm{N}=84)$ departments. But a higher percentage of infection was noticed in samples received from RICU (89 \%) followed by medicine (63\%) and emergency medicine (23\%) departments. 
Six hundred and seventy-three (79.4\%) samples showed culture positivity among 848 samples and the remaining 175 (20.6\%) samples were sterile. In our study, the most common organisms isolated were gram-negative bacilli which included Acinetobacter spp, Klebsiella spp, Pseudomonas, Escherichia spp and predominant being Acinetobacter spp. Summaiya et al. found that the most common organisms isolated in ETT were Pseudomonas Aeruginosa and Acinetobacter spp which is in similarity with our study. ${ }^{13}$ In Nusrat et al. study, the most common bacteria identified were Acinetobacter spp. (43.2 \%), Klebsiella spp. (20 $\%)$ and Pseudomonas spp. (18.9\%). ${ }^{14}$ Our study is concordant with Nusrath et al. study.

Patil et al. study noted that Pseudomonas aeruginosa was the most commonly isolated organism, followed by Klebsiella pneumoniae. ${ }^{15}$ In another study by George et al., Acinetobacter spp was the most common isolate (37.5 \%), followed by Pseudomonas spp (21.8\%) and Klebsiella spp (15.6\%) which is $50 \%$ concordant with our study. ${ }^{16}$

In our study, 186 (21.7\%) samples processed for culture positivity were polymicrobial and it could be due to multiple invasive procedures like artificial ventilation, central venous line insertion and catheterization. These procedures introduce multiple microbial floras which could have enhanced the chance of infection in debilitated patients. This was correlated with Siddique et al. and Malik et al. (20.3\%) studies. 17,18

In our study, $68 \%$ of organisms were multidrug-resistant. Among them, $60 \%$ were extended-spectrum beta-lactamase organisms, $48 \%$ were aminoglycoside resistant and $58 \%$ were carbapenem-resistant. None of the isolates was resistant to polymyxin.

More than $50 \%$ of Acinetobacter spp and Klebsiella spp were resistant to aminoglycosides and the precise percentage being $61.7 \%$ and $58 \%$ respectively. Increased resistance to fluoroquinolones was observed in Acinetobacter spp and Klebsiella spp i.e., $80 \%$ and $71 \%$ respectively. In our study, pan drug-resistant organisms were not at all isolated.

Various studies have observed the pattern of tracheal colonization and shown that over a period of time, the microorganisms gradually colonize the trachea. Candida spp were colonizers in the mouth, throat and trachea. Hamet et al. reported that association between multidrug-resistant organisms and candida spp and also underlying disease to be considered while reporting. Most of the gram-negative bacilli rapidly colonize their ways in mechanically ventilated patients and become pathogenic often. ${ }^{19}$

And colonization of bacteria depends on the conditions like the length of hospital stay, associated risk factors, infection control practices and environmental sanitation and prior antimicrobial therapy. 20

In our study, among gram-negative organisms, $98.9 \%$ were susceptible to polymyxin B, $96.3 \%$ were sensitive to tigecycline and $58 \%$ were sensitive to cefoperazonesulbactam. Among gram-positive organisms, vancomycin was the most sensitive antibiotic.

In the Sreeja Vamsi et al. study, carbapenem-resistant gram-negative bacilli organisms were $38 \%$, in Klebsiella spp $56 \%$, Pseudomonas spp $24 \%$, Escherichia spp $65 \%$ and Acinetobacter spp were $4.2 \%$. Carbapenem resistance in endotracheal samples was $25 \%$ and among them, carbapenem-resistant Enterobacteriaceae (CRE) was $68 \% .{ }^{21}$
In our study, the percentage of carbapenem resistance in GNB was $58 \%$, comparatively CRE was high and it might be depending on the hospital set up, length of stay in hospital, poor infection prevention and control practices implementation, lack of clinical application of antibiotic policy, overuse/ misuse of carbapenems and poor interaction between clinician and microbiologist. In our study, high carbapenem resistance among gram-negative bacilli was observed in Klebsiella spp which is showing similarity to the Sreeja Vamsi et al. study. A combination of colistin/polymyxin and tigecycline can be used to treat carbapenem-resistant gram-negative bacilli isolates.

Amid all gram-negative bacilli, 23 isolates were resistant to tigecycline and contributed $65.2 \%$ in Acinetobacter spp, $17.4 \%$ in Klebsiella spp, and $4.3 \%$ in Citrobacter spp. But tigecycline resistance could be due to genetic transfer. As tigecycline is a bacteriostatic antibiotic it cannot be used alone. That is why it should be given in combination with bactericidal antibiotics for better efficacy. In Ahmad et al. study, Acinetobacter spp carrying tigecycline resistance was $5.3 \%$. In our study, tigecycline resistance to Acinetobacter spp was $17.4 \%$ which is higher when compared to Ahmad et al. study. It could be due to sampling size variation, misuse of tigecycline, lack of knowledge and hospital settings.

Our study was showing concordant results with Ahmed $\mathrm{H}$ et al. study, the most common isolate being acinetobacter spp. However, tigecycline was found to be effective against Acinetobacter spp despite $17.4 \%$ of resistance in our study. ${ }^{22}$ Now it is high time to advance for cessation of antibiotic cycling for tigecycline as reported resistance was high in our setting.

The knowledge of antimicrobial therapy of locality and susceptibility pattern is essential to start empirical therapy as an initial treatment. Proper interaction and coordination are essential between clinician and microbiologist for escalation/de-escalation of antibiotic therapy. Institutional based antibiotic policy to be framed periodically and shall be available to all consultants to reduce sepsis-related morbidity and mortality.

\section{CONCLUSIONS}

The most common complication of mechanical ventilation in ICU is endotracheal infections particularly due to drugresistant microorganisms which will lead to prolonged stay of hospital, morbidity and mortality. These microorganisms could be exogenous or endogenous. Proper specimen collection and culture of tracheal secretions can permit proper diagnosis and improve the clinical outcome for these patients with the right antibiotic at right time in the correct dosage to avoid complications due to mechanical ventilation. If the patient is not treated early, the patient may succumb to ventilator-associated pneumonia. Hence it is essential to analyse the most common microbiome in endotracheal secretions, crucial to treat early as possible as per institutional antibiotic policy on an emergency basis and will be able to differentiate between true infection and colonization. The role of hospital infection prevention control has been increasing to eliminate the source of infection and to control the spread of infection by implementing proper hand hygiene tools in the daily practices of healthcare workers. 
The analysis of the microbiome and its susceptibility pattern must be updated regularly at least once in six months and to be clinically audited to choose proper antibiotics empirically in all ICU settings as part of diagnostic \& therapeutic antimicrobial stewardship.

Data sharing statement provided by the authors is available with the full text of this article at jemds.com.

Financial or other competing interests: None.

Disclosure forms provided by the authors are available with the full text of this article at jemds.com.

\section{REFERENCES}

[1] Horan TC, White JW, Jarvis WR, et al. Nosocomial infection surveillance, 1984. MMWR CDC Surveill Summ 1986;35(1):17-29.

[2] Morehead RS, Pinto SJ. Ventilator-associated pneumonia. Arch Intern Med 2000;160(13):1926-36.

[3] Hess DR. Approaches to conventional mechanical ventilation of the patient with acute respiratory distress syndrome. Respir Care 2011;56(10):1555-72.

[4] Craven DE, Chroneou A, Zias N, et al. Ventilatorassociated tracheobronchitis: the impact of targeted antibiotic therapy on patient outcomes. Chest 2009;135(2):521-8.

[5] Bonten MJM, Kollef MH, Hall JB. Risk factors for ventilator-associated pneumonia: from epidemiology to patient management. Clin Infect Dis 2004;38(8):1141-9.

[6] Gardland A. Improving the ICU: part 2. Chest 2005;127(6):2165-79.

[7] Center for Disease Control and prevention. (2019). National Healthcare Safety Network. Available from https://www.cdc.gov/nhsn/index.html/.

[8] Cavalcanti M, Valencia M, Torres A. Respiratory nosocomial infections in the medical intensive care unit. Microbes Infect 2005;7(2):292-301.

[9] Winn WC, Koneman EW. Koneman's color atlas and textbook of diagnostic microbiology. $6^{\text {th }}$ edn. Philadelphia: Lippincott Williams and Wilkins 2006:167.

[10] Mackie TJ, McCartney JE. Mackie \& McCartney practical medical microbiology. $18^{\text {th }}$ edn. New York: Churchill Livingstone 1996:151-69.

[11] Clinical and Laboratory Standards Institute (CLSI). Performance Standards for Antimicrobial Susceptibility Testing, CLSI document M100. 30th edn. Wayne, PA: Clinical and Laboratory Standards Institute 2019.

[12] Chandra D, Laghawe A, Sadawarte $K$, et al. Microbiological profile and antimicrobial sensitivity pattern of endotracheal tube aspirates of patients in ICU of a tertiary care hospital in Bhopal. Int J Curr Microbiol App Sci 2017;6(3):891-5.

[13] Summiya MA, Urmi NJ. Assessment of biofilms formation by the causative organisms of ventilator associated pneumonia at intensive care unit of a tertiary care hospital. Natl J Med Res 2012;2(1):15-8.

[14] Nusrat $T$, Akter N, Rahman NAA, et al. Antibiotic resistance and sensitivity pattern of Metallo- $\beta$ Lactamase Producing Gram-Negative Bacilli in ventilator-associated pneumonia in the intensive care unit of a public medical school hospital in Bangladesh. Hosp Pract 2020;48(3):128-36.

[15] Patil T. The study of the organisms colonising trachea in mechanically ventilated patients admitted in the intensive care unit (ICU). Int J Med Sci Educ 2014;1(1):39-47.

[16] George P, Sequiera A. Antimicrobial sensitivity pattern among organisms isolated from the endotracheal aspirates of patients with ventilator associated pneumonia. J Clin Diagn Res 2010;4:3397-401.

[17] Siddique SG, Bhalchandra MH, Wyawahare AS, et al. Prevalence of MRSA, ESBL and Carbapenemase producing isolates obtained from endotracheal and tracheal tubes secretions of ICU patient at tertiary care center. Int J Curr Microbiol App Sci 2017;6(4):288-99.

[18] Malik M, Malik MI, Sajjad A. Pattern of bacterial pathogens isolated from endotracheal secretions in Intensive care unit (ICU) patients of a tertiary care hospital of Lahore. Pak J Pathol 2018;29(2):46-8.

[19] Hamet M, Pavon A, Dalle F, et al. Candida spp. airway colonization could promote antibiotic resistant bacteria selection in the patients with suspected ventilator associated pneumonia. Intensive Care Med 2012;38(8):1272-9.

[20] Garrouste-Orgeas G, Chevret S, Arlet G, et al. Oropharyngeal or gastric colonization and nosocomial pneumonia in adult intensive care unit patients. A prospective study based on genomic DNA analysis. American Journal of Respiratory and Critical Care Medicine 1997;156(5):1647-55.

[21] Vamsi KS, Ramamoorthy S, Murali TS, et al. Prevalence of carbapenem resistant gram negative bacteria in rural Hospital Mahabubnagar, Telangana and Systemic Review. Int J Curr Microbiol App Sci 2021;10(3):1542-7.

[22] Ahmad H, Sadiq A, Bhatti HW, et al. Bacteriological profile and antibiogram of cultures isolated from tracheal secretions. Cureus 2019;11(6):e4965. 